\title{
On Determining the Location of Complex Zeros of Solutions of Certain Linear Differential Equations (*).
}

\author{
STEVEN B. BANK \\ Summary. - We investigate the location of zeros of solutions for a class of second-order linear \\ differential equations. This class had previously been investigated to determine the frequency \\ of zeros of solutions AMS(MOS): 34A20.
}

\section{1. - Introduction.}

Several recent papers (e.g., [6] and [7]) have dealt with the problem of determining the frequency of zeros of solutions of certain classes of linear differential equations of the form,

$$
w^{\prime \prime}+A(z) w=0
$$

where $A(z)$ is an entire function. For example, it was shown in [6] that if $A(z)$ is of the form $\sum_{j=1}^{n} B_{j} e^{P_{j}}$, where $P_{1}, \ldots, P_{n}$ are nonconstant polynomials of degrees $d_{1}, \ldots, d_{n}$ respectively, such that $(a) P_{i}-P_{j}$ is of degree $\max \left\{d_{i}, d_{j}\right\}$ if $i \neq j$, and (b) $B_{j} \neq 0$ is an entire function of order less than $d_{j}$ for $j=1, \ldots, n$, then at least one of any two linearly independent solutions of (1) has the property that the exponent of convergence of its zero-sequence is infinite. (The same conclusion also holds if $A(z)$ is of the form $\sum_{j=1}^{n} B_{j} e^{P_{j}}+Q$, where the $P_{j}$ and $B_{j}$ satisfy $(a)$ and $(b)$ above, and where $Q$ is a polynomial whose degree $m$ satisfies $(m+2) / 2<\max \left\{d_{i}\right\}$.) More recently, it was shown in [7] that when $A(z)$ is of the form $B e^{P}+Q$, where $B$ $P$, and $Q$ are polynomials such that $B \neq 0$, and for which the degrees $m$ and $n$ of $P$ and $Q$ respectively satisfy $n<2(m-1)$, then every solution $w \neq 0$ of (1) has the property that the exponent of convergence of its zero-sequence is infinite. (Other related results can be found in [1] and [5].)

(*) Entrata in Redazione il 18 aprile 1987.

This research was supported in part by the National Science Foundation (DMS 84-20561).

Indirizzo dell'A.: Department of Mathematies, University of Illinois, 1409 West Green Street, Urbana, Illinois 61801, U.S.A. 
In view of the above results, it is natural to ask for the regions where the zeros of solutions are located, and our main results address this question for those equations (1) where $A(z)$ is of the form,

$$
A(z)=\sum_{j=q}^{m} Q_{j}(z) e^{j P(z)}
$$

where $q$ and $m$ are any integers (positive, negative, or zero), where the $Q_{j}(z)$ are polynomials, and where $P(z)$ is any noneonstant polynomial. We remark that in the case where $A(z)$ in (1) is a polynomial, there is a classical result due jointly to E. Hille, R. Nevanlinna, and H. Wituich (see [16; p. 282]), which determines the location of zeros of the solutions of (1). By a linear change of independent variable, one can assume that the leading term in $A(z)$ is $-((n+2) / 2)^{2} z^{n}$. Then the Hille-Nevanlinna-Wittich result states that for any solution $f(z) \neq \equiv$ of $(1)$, and any $\varepsilon>0$, all but finitely many zeros of $f$ lie in the union of the sectors,

$$
\left|\arg z-\pi(2 j+1)(n+2)^{-1}\right|<\varepsilon,
$$

for $j=0,1, \ldots, n+1$. This result was proved by using a method of asymptotic integration (see $[10 ;$ Chapter $7, \S 4]$ or $[11 ;$ p. 345]) to construct in each of the closed sectors $J$ which lies between two adjacent sectors in (3), a fundamental set $\left\{f_{1}, f_{2}\right\}$ of solutions of (1), each having only finitely many zeros in $\mathcal{J}$, and having the property that $f_{1} / f_{2} \rightarrow \infty$ as $z \rightarrow \infty$ in $J$. Then clearly any nontrivial linear combination of $f_{1}$ and $f_{2}$ can have only finitely many zeros in $J$. (In fact, the solutions $f_{1}, f_{2}$ which are constructed satisfy the asymptotic relation, $\log f_{j} \sim \pm z^{(n+2) / 2}$ as $z \rightarrow \infty$ in $J$.)

In view of the above result, we can assume that $A(z)$ in (2) is transcendental. Clearly we may also assume in (2) that,

$$
q \leqslant m, \quad Q_{m} \neq 0 \quad \text { and } \quad Q_{q} \neq 0
$$

If the leading term of the polynomial $P(z)$ in (2) is $a_{n} z^{n}$, we observe that the behavior of $e^{P(z)}$ allong a ray $\arg z=\varphi$ is determined by the sign of the function $\Delta(P, \varphi)$ defined by,

$$
\Delta(P, \varphi)=\operatorname{Cos}\left(n \varphi+\arg a_{n}\right)
$$

since,

$$
\left|e^{a_{n} \tilde{z}^{n}}\right|=e^{\Delta(P, \varphi)\left|\alpha_{n}\right||z| n}
$$

Letting $\varphi_{0}$ be any zero of $\Delta(P, \varphi)$, it is easy to see that the set of zeros of $\Delta(P, \varphi)$ in the closed interval $\left[\varphi_{0}, \varphi_{0}+2 \pi\right]$ consists of the points $\varphi_{0}<\varphi_{1}<\ldots<\varphi_{2 n}$, where $\varphi_{j}=\varphi_{0}+(j \pi / n)$. For each pair $\left(\varphi_{j}, \varphi_{i_{+1}}\right)$ of consecutive zeros of $\Delta(P, \varphi)$, and any 
$\varepsilon>0$, our main result will be concerned with the location of the zeros of a solution of (1) in the sector,

$$
\varphi_{j}+\varepsilon<\arg z<\varphi_{j_{+1}}-\varepsilon
$$

Our first step is to perform a rotation, $s=z e^{-i \varphi_{j}}$, which maips the sector,

$$
S_{j}: \varphi_{j}<\arg z<\varphi_{j+1},
$$

onto the sector,

$$
S: 0<\operatorname{Arg} s<\pi / n \text {. }
$$

(As is customary, we will denote by Arg $s$ and $\log s$, the principal branches of these functions in $|\operatorname{Arg} s|<\pi$. On the same domain, we will denote by $s^{\beta}$, the function $\exp (\beta \log s)$ for any complex number $\beta$.) Thus, for any solution $w(z)$ of (1) defined on the sector $S_{j}$ in (8), we define $u(s)$ on the sector $S$ in (9) by,

$$
u(s)=w\left(s e^{i \varphi_{j}}\right),
$$

so that $u(s)$ satisfies the equation,

$$
u^{u}(s)+\left(\sum_{j=q}^{m} R_{j}(s) e^{j T(s)}\right) u(s)=0,
$$

where $R_{j}(s)=e^{2 i \varphi_{j}} Q_{j}\left(s e^{i \varphi_{j}}\right)$, and where $T(s)=P\left(s e^{i \varphi_{j}}\right)$. Thus, the $R_{j}(s)$ are polynomials in $s$, and clearly $T(s)$ is a polynomial in $s$ whose leading term is $a_{n} e^{i n \varphi_{s}} s^{n}$. Since $\Delta\left(P, \varphi_{j}\right)=0$ (where $\Delta$ is given in (5)), it is easy to see that the leading coeffcient of $T(s)$ is purely imaginary, so that

$$
T(s)=i b s^{n}+\ldots, \quad \text { where } b \text { is real } .
$$

(We note that if $\Delta(P, \varphi)>0$ on $\left(\varphi_{j}, \varphi_{j+1}\right)$ then $b<0$, while if $\Delta(P, \varphi)<0$ on $\left(\varphi_{i}, \varphi_{j_{+1}}\right)$, then $b>0$. This is easy to see from the relation,

$$
b=\operatorname{Im}\left(a_{n} e^{i n \varphi_{j}}\right)=-\left(\left|a_{n}\right| / n\right) \Delta^{\prime}\left(P, \varphi_{j}\right),
$$

where the prime denotes differentiation with respect to $\varphi$.)

Hence, in view of (11) and (12), it suffices to consider the location of zeros on the sector $S: 0<\operatorname{Arg} z<\pi / n$, of a solution $w(z) \neq \equiv 0$ of our original equation (1), where $A(z)$ is given by $(2)$, and where the polynomial $P(z)$ has the form,

$$
P(z)=i b z^{n}+a_{n-1} z^{n-1}+\ldots, \quad \text { where } b \text { is real },
$$


and where the $Q_{j}$ are polynomials for which (4) holds. Of course, the asymptotic behavior of $A(z)$ along rays in $S$ depends not only on the sign of $b$ in (14) but also on the signs of the integers $m$ and $q$ in (2). Initially, we divide all the possibilities into the following six cases: (i) $0 \leqslant q \leqslant m, b>0$; (ii) $0 \leqslant q \leqslant m, b<0$; (iii) $q \leqslant m \leqslant 0$, $b>0$; (iv) $q \leqslant m \leqslant 0, b<0$; (v) $q \leqslant 0 \leqslant m, b>0$; (vi) $q \leqslant 0 \leqslant m, b<0$. However recalling that we are assuming that $A(z)$ in (2) is not a polynomial, we now show that by replacing $P$ by $-P$ in (2) if necessary, it suffices to consider only the following two cases $(A)$ and $(B):(A) m>0$ and $b<0$, and $(B) 0 \leqslant q \leqslant m$ and $b>0$. (To see this, we note first that (i) is $(B)$. In case (ii), we note that $m>0$ since $A(z)$ is transcendental, so (ii) falls under $(A)$. In (iii), we must have $q<0$, and so we can rewrite (2) as,

$$
A(z)=\sum_{k=-m}^{-\alpha} Q_{-k}(z) e^{k P_{1}(z)}, \quad \text { where } P_{1}=-P,
$$

which clearly falls under case $(A)$. In (iv), we rewrite $A(z)$ as in (15), and it is covered by $(B)$. In (v), we have $(B)$ if $q=0$, while in the case $q<0$, we obtain $(A)$ when $A(z)$ is written in the form (15). Finally, in (vi), we have $(A)$ if $m>0$, while if $m=0$, we have $(B)$ when $A(z)$ is rewritten in the form (15).) Our two main results which now follow (and which, will be proved in $\$ \S 3$ and 5), consider the two cases $(A)$ and $(B)$ respectively.

Theorem 1. - Let $A(z)$ be an entire transcendental function of the form (2), where $(a) P(z)$ is a nonconstant polynomial of the form (14) for some constant $b<0 ;(b) q$ and $m$ are integers with $q \leqslant m$ and $m>0 ;(c) Q_{q}, Q_{q+1}, \ldots, Q_{m}$ are polynomials with $Q_{q} \neq 0$ and $Q_{m} \neq \equiv$, say $Q_{m}(z)=d_{\beta} z^{\beta}+d_{\beta-1} z^{\beta-1}+\ldots$, where $d_{\beta}$ is a nonzero constant. Define real numbers $\alpha, \theta_{0}, \ldots, \theta_{m}$ as follows:

$$
\begin{aligned}
& \alpha=-(2 / m)\left((m \pi / 2)+((\beta+2) \pi / 4 n)+\left(\arg d_{\beta}\right) / 2\right), \\
& \theta_{j}=-\pi+(2 j \pi / m) \quad \text { for } j=0,1, \ldots, m .
\end{aligned}
$$

For any real numbers $\varepsilon>0$ and $\delta>0$, and any integers $p$ and $j$ with $0 \leqslant j \leqslant m-1$, let $A_{\varepsilon \delta y j}$ denote the set of all points $z$ in the sector $\varepsilon<\operatorname{Arg} z<(\pi / n)-\varepsilon$ for which

$$
\theta_{j}+\alpha+2 \pi p+\delta<\operatorname{Im} P(z)<\theta_{j+1}+\alpha+2 \pi p-\delta .
$$

Then, any solution $w(z) \neq \equiv$ of $(1)$ can have at most finitely many zeros in any $\Lambda_{\varepsilon \delta p j}$.

THEOREM 2. - Let $A(z)$ be an entire transcendental function of the form (2) where $(a) P(z)$ is a nonconstant polynomial of the form (14) for some constant $b>0$; (b) $q$ and $m$ are any integers with $0 \leqslant q \leqslant m ;(c) Q_{q}, \ldots, Q_{m}$ are polynomials with $Q_{a} \neq \equiv 0$ and $Q_{m} \neq \equiv 0$. Then:

(I) If $q>0$, then for any $\varepsilon>0$, any solution $w(z) \neq \equiv$ of (1) can bave at most finitely many zeros in the sector, $\varepsilon<\operatorname{Arg} z<(\pi / n)-\varepsilon$. 
(II) If $q=0$, let $Q_{0}(z)=c_{\lambda} z^{\lambda}+c_{\lambda-1} z^{\lambda-1}+\ldots$, where $c_{\lambda} \neq 0$, and let $H(\theta)$ denote the function,

$$
H(\theta)=\operatorname{Cos}\left(\frac{1}{2}\left((\lambda+2) \theta+\arg \left(-c_{\lambda}\right)\right)\right)
$$

If $H(\theta)$ has any zeros on $(0, \pi / n)$, let these zeros be $\psi_{1}<\psi_{2}<\ldots<\psi_{N}$ where $N \geqslant 1$. Set $\psi_{0}=0$ and $\psi_{N+1}=\pi / n$. (If $H(\theta)$ has no zeros on $(0, \pi / n)$, set $N=0$ so that $\psi_{0}=0$ and $\left.\psi_{1}=\pi / n.\right)$ Then, for any $\varepsilon>0$, any solution $w(z) \neq 0$ of $(1)$ can have at most finitely many zeros in the sector

$$
\psi_{j}+\varepsilon<\operatorname{Arg} z<\psi_{j_{+1}}-\varepsilon
$$

for each $j=0,1, \ldots, N$.

For the type of equation considered in Theorem 1, it follows from Theorem 1 that if a solution $w(z) \neq 0$ of (1) has infinitely many zeros in that part of the sector $\varepsilon<\operatorname{Arg} z<(\pi / n)-\varepsilon$ (for some $\varepsilon>0$ ) for which $a \leqslant \operatorname{Im} P(z) \leqslant c$, where $a$ and $c$ are any real numbers with $a<c$, then these zeros must be "concentrated " (in a certain sense) around a finite number of special curves of the form $\operatorname{Im} P(z)=K$, where $K$ is a value of $\theta_{j}+\alpha+2 \pi p$. In the special case where $P(z)$ is a linear polynomial (which can be taken to be $i b z$ by incorporating any constant term into the $Q_{j}$ ), these curves are the vertical lines $x=K / b$. We remark that the special case where $P(z)$ is linear, and the $Q_{j}$ are constant polynomials was treated in an earlier paper [4] on periodic equations, and an analogous result to Theorem 1 was proved in this special case. Two illustrative examples were given in [4], namely,

$$
w^{\prime \prime}+\left(e^{z}-(1 / 16)\right) w=0
$$

and Mathieu's equation,

$$
w^{\prime \prime}+(a(\operatorname{Cos} 2 z)+c) w=0,
$$

where $a$ and $e$ are complex constants, with $a \neq 0$. For $(21)$, the function $\Delta(P, \varphi)$ given in (5) is just $\operatorname{Cos} \varphi$ with zeros at $\varphi_{0}=-\pi / 2, \varphi_{1}=\pi / 2$, and $\varphi_{2}=3 \pi / 2$ in $[-\pi / 2,3 \pi / 2]$. After performing the rotation (10) of the sector $S_{0}$ in (8) into the sector $S$ in (9), the resulting equation is covered by Theorem 1. After applying Theorem 1, and retransforming back to the right half-plane, we find that any for any solution of (21) which has infinitely many zeros in a closed horizontal semiinfinite strip in the right half-plane, these zeros must be concentrated around the horizontal lines $y=2 \pi p$, where $p$ is an integer. To check the sharpness of this result, we remark that it was known (see [5; $\$ 5(b)]$ ) that $(21)$ could be explicitely solved, and possesses two linearly independent solutions $f_{1}$ and $f_{2}$, each having no zeros. By explicit calculation, it was shown in [4] that the zeros of any linear combination $\alpha_{1} f_{1}+\alpha_{2} f_{2}$, (where $\alpha_{1}$ and $\alpha_{2}$ are both nonzero constants), in a closed hori- 
zontal semi-infinite strip in the right half-plane, are indeed concentrated around the lines $y=2 \pi p$. For the other sector $S_{1}$ in (8) (i.e. the left half-plane), the rotation (10) leads to an equation which is covered by Theorem 2 , and we find that any solution $w(z) \neq \equiv 0$ of $(21)$ can have at most finitely many zeros in the sector,

$$
(\pi / 2)+\varepsilon<\arg z<(3 \pi / 2)-\varepsilon,
$$

for any $\varepsilon>0$. Again, this agrees sharply with the explicit calculation of the zeros. We further remark that the explicit calculation for (21) shows that all solutions $\alpha_{1} f_{1}+\alpha_{2} f_{2}$ of (21) where $\alpha_{1}$ and $\alpha_{2}$ are both nonzero, have infinitely many zeros in both of the sectors,

$$
|\operatorname{Arg} z-(\pi / 2)|<\varepsilon \quad \text { and } \quad|\operatorname{Arg} z+(\pi / 2)|<\varepsilon
$$

(where $\varepsilon>0$ is arbitrary). These sectors surround the two rays $\operatorname{Arg} z= \pm(\pi / 2)$ where $e^{z}$ changes behavior, and these zeros cannot be predicted by our results.

In the case of Mathieu's equation (22), it was shown in [4] that the results predicted for the upper half-plane and lower half-plane (both being covered by Theorem 1), were in agreement with the results obtained in the special case where $a$ and $c$ are real constants by $\mathrm{E}$. HILLE in [8].

In the case where $P(z)$ in (2) is a quadratic polynomial, it is easy to see that by completing the square in $P(z)$ (and incorporationg the constant term into the $Q_{j}$ ), that under a translation, the equation (1) can be transformed to an equation where the only term in $P(z)$ is of degree 2. Thus when the rotation (10) is performed on any $S_{j}$ given by $(8)$ for which $\Delta(P, \varphi)>0$ on $\left(\varphi_{j}, \varphi_{j+1}\right)$, we obtain an equation (1) where in (2) we have $P(z)=i b z^{2}$ for some $b<0$. In this case, each of the special curves $\operatorname{Im} P(z)=K$ mentioned earlier, around which the zeros are concentrated, is part of the hyperbola $b\left(x^{2}-y^{2}\right)=K$, except in the case $K=0$, when we obtain the ray $\operatorname{Arg} z=\pi / 4$. It is easy to see that there are examples where this ray is indeed the location of infinitely many zeros of certain solutions. For example, if we begin with an equation (1) where in (2) we assume that $m \geqslant q \geqslant 0$, and that the polynomials $Q_{j}$ and $P$ have positive coefficients, with $P(z)$ of the form $d z^{n}$ where $n$ is arbitrary, then the Sturm comparison theorem or Wiman's formula (see [10; p. 472]) shows that any real-valued solution of (1) on the positive real-axis has zeros on this axis with infinite exponent of convergence. When the rotation (10) is performed on the sector (8) with $\varphi_{j}=-\pi / 2 n$ and $\varphi_{j_{+1}}=\pi / 2 n$, to obtain the sector (9) and the equation (11), the positive real axis is transformed to Arg $s=\pi / 2 n$. Of course, this ray will be predicted by Theorem 1, since it is easy to see that for the transformed equation, we obtain $\alpha=-\pi$ in (16), and so the special curve, $\operatorname{Im} T(s)=\theta_{j}+\alpha+2 \pi p$ reduces to $\operatorname{Im} T(s)=0$ when $j=m$ and $p=0$. But in the transformed equation, $T(s)$ has the form $-i d s^{n}$, and hence the points on the ray $\operatorname{Arg} s=\pi / 2 n$ lie on $\operatorname{Im} T(s)=0$. 
In our final result (to be proved in $\S 6$ ) we show that for the type of equation considered in Theorem 1 , each of the special curves, $\operatorname{Im} P(z)=\theta_{j}+\alpha+2 \pi p$, has the property that there are always certain solutions of equation (1) which have infinitely many zeros around it. Similarly, for those equations treated in Theorem 2, Part (II), we show that each of the exceptional rays $\operatorname{Arg} z=\psi_{j}$, where $1 \leqslant j \leqslant N$, has the property that there are always solutions of (1) which have infinitely many zeros in arbitrarily small sectors around it. We prove:

THeorem 3. - (A) Assume the hypothesis and notation of Theorem 1, and let $a_{j p}$ be any value of $\theta_{j}+\alpha+2 \pi p$ for some integers $p$ and $j$ with $0 \leqslant j \leqslant m$. Let $\varepsilon_{1}$ and $\delta_{1}$ be any positive real numbers with $\varepsilon_{1}<\pi / 2 n$. Then, there exists a fundamental set, $w_{1}$ and $w_{2}$, of solutions of (1) with the property that any linear combination $c w_{1}+c_{0} w_{2}$, where the complex constants $e$ and $c_{0}$ are both nonzero, has zeros with infinite exponent of convergence in that part of the sector $\varepsilon_{1}<\operatorname{Arg} z<(\pi / n)-\varepsilon_{1}$ for which $a_{j p}-\delta_{1}<\operatorname{Im} P(z)<a_{j p}+\delta_{1}$.

(B) Assume the hypothesis and notation of Theorem 2, and assume $q=0$ and $N \geqslant 1$. Then for any $\varepsilon_{1}>0$ and any integer $j$, with $1 \leqslant j \leqslant N$, there exists a fundamental set, $w_{1}$ and $w_{2}$, of solutions of (1) with the property that any linear combination $c w_{1}+c_{0} w_{2}$, where the complex constants $c$ and $c_{0}$ are both nonzero, has zeros with exponent of convergence at least $(\lambda / 2)+1$ in the sector $\psi_{j}-\varepsilon_{1}<$ $<\operatorname{Arg} z<\psi_{j}+\varepsilon_{1}$.

The key tool in the proofs of Theorems 1, 2 and 3 are asymptotic existence theorems which were proved by W. STRoDT. The Strodt theory which was developed in [12] treats first-order differential equations of the form,

$$
\Omega\left(\zeta, u, u^{\prime}\right)=h(\zeta)
$$

where $\Omega$ is a polynomial in $u$ and $u^{\prime}$, all of whose terms have strictly positive total degree, and whose coefficients, together with $h(\zeta)$, are analytic functions of $\zeta$ in a sectorial region $D$, which possess asymptotic expansions as $\zeta \rightarrow \infty$ in terms of functions (called logarithmic monomials) which are of the form,

$$
K \zeta^{a_{0}}(\log \zeta)^{a_{1}}(\log (\log \zeta))^{a_{a}} \ldots\left(\log _{p} \zeta\right)^{a_{p}}
$$

where the $a_{j}$ are real, and $K \neq 0$ is complex. The essential concept in [12] is that of principal monomial of (25), which is defined as any logarithmic monomial $M(\zeta)$ with the following two properties: (i) $\Omega\left(\zeta, M(\zeta), M^{\prime}(\zeta)\right)$ is asymptotically equivalent to $h(\zeta)$ as $\zeta \rightarrow \infty$ in $D$, and (ii) for any logarithmic monomial $N(\zeta)$ which is $o(M(\zeta))$, we have $\Omega\left(\zeta, N(\zeta), N^{\prime}(\zeta)\right)=o(h(\zeta))$ as $\zeta \rightarrow \infty$ in $D$. StRoDt [12; p. 28] developed an algorithm based on a Newton polygon construction, which produces the set of principal monomials of a given equation in a finite number of steps, and it was 
shown in [12; p. 72], under general conditions on $\Omega$ and $h$, that corresponding to any principal monomial $M$, there exist one or more solutions of (25) in sectorial subregions of $D$, which are asymptotically equivalent to $M$ as $\zeta \rightarrow \infty$. (Such a solution is called a principal solution of (25). We remark that extensions of these results to higher-order equations, and to other logarithmic monomials can be found in [2], [13] and [15].)

In this paper, we will require Strodt's results in the simple case of a Riccati equation. When such results are required, we will explain all the relevant terminology and notation (see § 2 ).

\section{2. - Concepts from the Strodt theory.}

(a) $[12 ; \S 94]$ : The neighborhood system $F(a, b)$. Let $-\pi \leqslant a<b \leqslant \pi$. For each nonnegative real-valued function $g$ on $(0,(b-a) / 2)$, let $V(g)$ be the union (over all $\delta \in(0,(b-a) / 2))$ of all sectors, $a+\delta<\operatorname{Arg}(\zeta-h(\delta))<b-\delta$, where $h(\delta)=$ $=g(\delta) e^{i(a+b) / 2}$. The set of all $\nabla(g)$ (for all choices of $g$ ) is denoted $F(a, b)$, and is a filter base which converges to $\infty$. Each $V(g)$ is a simply-connected region (see [12; $\S 93]$ ), and we require the following simple fact:

Lemra A. - Let $V$ be an element of $F(a, b)$, and let $\varepsilon>0$ be arbitrary. Then there is a constant $R_{0}(\varepsilon)>0$ such that $V$ contains the set,

$$
a+\varepsilon \leqslant \operatorname{Arg} \zeta \leqslant b-\varepsilon, \quad|\zeta| \geqslant R_{0}(\varepsilon) .
$$

Proof. - Let $g$ be a nonnegative real-valued function on $(0,(b-a) / 2)$ for which $V=V(g)$. Then $V(g)$ contains a sector of the form, $a+\delta<\operatorname{Arg}\left(\zeta-\zeta_{0}\right)<b-\delta$, where $0<\delta<\varepsilon$ and $\operatorname{Arg} \zeta_{0}=(a+b) / 2$, and it is obvious by geometric reasoning that the sides of this sector must intersect the corresponding sides of the sector $a+\varepsilon \leqslant \operatorname{Arg} \zeta \leqslant b-\varepsilon$. The lemma now follows immediately.

(b) $[12 ; \S 13]$ : The relation of asymptotic equivalence. If $f(\zeta)$ is an analytic function on some element of $F(a, b)$, then $f(\zeta)$ is called admissible in $F(a, b)$. If $e$ is a complex number, then the statement $f \rightarrow c$ in $F(a, b)$ means (as is customary) that for any $\varepsilon>0$, there exists an element $V$ of $F(a, b)$ such that $|f(\zeta)-c|<\varepsilon$ for all $\zeta \in V$. The statement $f \ll 1$ in $F^{\prime}(a, b)$, means that in addition to $f \rightarrow 0$, all the functions $\theta_{j}^{k} f \rightarrow 0$ in $F(a, b)$, where $\theta_{j}$ denotes the operator

$$
\theta_{i} f=\zeta(\log \zeta) \ldots\left(\log _{j-1} \zeta\right) f^{\prime}(\zeta)
$$

and where (for $k \geqslant 0$ ), $\theta_{j}^{k}$ is the $k$-th iterate of $\theta_{j}$. The statements $f_{1} \ll f_{2}$ and $f_{1} \sim f_{2}$ in $F(a, b)$ mean respectively $f_{1} / f_{2} \ll 1$ and $f_{1}-f_{2} \ll f_{2}$. (This strong relation of asymptotic equivalence is designed to ensure that if $M$ is a non-constant logarithmic monomial, then $f \sim M$ implies $f^{\prime} \sim M^{\prime}$ in $F(a, b)$. See [12; §28].) Finally, if $f$ is 
admissible in $F(a, b)$, we will denote by $\int f$, any primitive of $f$ in an element of $F(a, b)$. We will require the following facts:

Lemar B. - (a) Let $h \sim c \zeta^{-1}$ in $F(a, b)$ for some nonzero complex constant $c$, and let $f=\exp \int h$. Then for all $\varepsilon>0, f \ll \zeta^{c+\varepsilon}$ in $F(a, b)$.

(b) Let $t>0$, and assume $g \ll \zeta^{-1-t}$ in $F(a, b)$. Let $f=\exp \int g$. Then, there exists a nonzero constant $e$ such that $f \rightarrow e$ in $F(a, b)$. (Of course, $c$ depends on which primitive of $g$ is chosen.)

(c) Let $M(\zeta)$ be a logarithmic monomial of the form (26) with $a_{0}>-1$, say $a_{0}=-1+t$ where $t>0$. Define $I(M, \varphi)=\operatorname{Cos}(t \varphi+\arg K)$ for $-\pi<\varphi<\pi$. (We will call $I(M, \varphi)$ the indicial function for $M(\zeta)$.) Let $u \sim M$ in some $F(a, b)$. Then, if $\left(a_{1}, b_{1}\right)$ is any subinterval of $(a, b)$ on which $I(M, \varphi)>0$ (respectively, $\left.I(M, \varphi)<0\right)$, then for all real $\beta$, we have $\exp \int u \gg \zeta^{\beta}$ (respectively, $\exp \int u \ll \zeta^{\beta}$ ) in $F\left(a_{1}, b_{1}\right)$.

Proof. - Part $(a)$ is proved in [3; Lemma $8(b)]$. Part $(b)$ is proved in [4; Section 2]. Part $(c)$ is proved in [3; Lemma 4] when $I(M, \varphi)>0$. The proof when $I(M, \varphi)<0$ follows by setting $M_{1}=-M$ and noting that $I\left(M_{1}, \varphi\right)>0$.

(c) $[14 ;$ p. 244]. Logarithmic fields. Let $p$ be a nonnegative integer. A function of the form (26) for some complex constant $K \neq 0$, and real constants $a_{i}$, is called a logarithmic monomial of rank $\leqslant p$. The set of all logarithmic monomials of rank $\leqslant p$ will be denoted $\Phi_{p}$. A logarithmic field of rank $p$ over $F(a, b)$ is a set $\Gamma$ of functions, each defined and admissible in $F(a, b)$, with the following properties: (i) $\Gamma$ is a field (where, as usual, we identify two elements of $\Gamma$ if they agree on an element of $F(a, b))$; (ii) $\Gamma$ contains $\Phi_{p}$; (iii) for every element $f$ in $\Gamma$ except zero, there exists $M$ in $\Phi_{p}$ such that $f \sim M$ over $F(a, b)$. (The simplest example of such a field is the set of rational combinations of the elements of $\Phi_{p}$. We will denote this field by $\Psi_{p}$.) We will require the following fact:

Lemma C. - Let $G(z, v)=\sum_{j=0}^{n} f_{j} v^{j}$ be a polynomial in $v$ of degree $n \geqslant 1$, whose coefficients $f_{0}, \ldots, f_{n}$ are elements of a logarithmic field of rank $p$ over $F(a, b)$. Then there exists a logarithmic field $\Gamma_{p}$ of rank $p$ over $F(a, b)$, in which $G(z, v)$ factors completely.

Proof. - This result follows immediately from [14; Theorem II, p. 244] by applying this result to, in the terminology of $[14 ;$ p. 246], the logarithmic quadruple $\left(F, E_{0}^{*}(F), R, S_{p}\right)$, where $F=F(a, b)$ and $R$ is the set of real numbers.

3. - Proof of Theorem 1. - We assume the hypothesis of the theorem, so that $P(z)$ has the form (14) for some $b<0$. We consider the field $\Psi_{0}$ (introduced in $\S 2(c)$ ) of all rational combinations of logarithmic monomials $K t^{a_{0}}$ of rank $\leqslant 0$, in a complex 
variable $t$. As noted in $\S 2(c), \Psi_{0}$ is a logarithmic field of rank zero over $F(-\pi, \pi)$, and clearly $\Psi_{0}$ contains the coefficients of the polynomial in $v$ defined by $P(v)-t$. Thus by Lemma $\mathrm{C}$, there exists a logarithmic field $\Gamma_{0}$ of rank zero over $F(-\pi, \pi)$. in which $P(v)-t$ factors completely. Hence there exist elements $u_{0}(t), \ldots, u_{n-1}(t)$ in $\Gamma_{0}$ such that,

$$
P(v)-t=i b\left(v-u_{0}(t)\right) \ldots\left(v-u_{n-1}(t)\right),
$$

for all functions $v=v(t)$ admissible in $F(-\pi, \pi)$. By condition (iii) for a logarithmic field of rank zero, it follows that for each $j=0,1, \ldots, n-1$, either $u_{j} \equiv 0$ or $u_{j} \sim M_{j}$ over $F(-\pi, \pi)$, where $M_{j}$ is a logarithmic monomial in $t$ of rank $\leqslant 0$. From (27), clearly $u_{j} \neq \equiv$ for each $j$. Since each $M_{j}$ is asymptotically equivalent to an exact solution of the equation $P(v)-t=0$, it follows [2; Lemma $5(c), p .87]$ that $M_{j}$ is a "critical monomial " of $P(v)-t$ as defined in [2; Section 4]. (In the terminology of [14; Section 5], the $M_{j}$ are "points of instability " for $P(v)-t$.) There is an algorithm in [2; Sections 21 and 26] which produces all the critical monomials of any differential polynomial with coefficients in a logarithmic field of arbitrary rank. (There is also a related algorithm in [14; Lemma 28, p. 236] which produces all the points of instability of any algebraic polynomial with coefficients in a logarithmic field.) Applying either algorithm, we find that the set of points of instability of $P(v)-t$ can be written in the form, $\sigma_{j} \sigma t^{1 / n}$ for $j=0,1, \ldots, n-1$, where

$$
\sigma=|b|^{-1 / n} e^{i \pi / 2 n}, \quad \text { and } \quad \sigma_{j}=e^{2 \pi i j / n} .
$$

Since the partial derivative with respect to $v$ of $P(v)-t$ is $P^{\prime}(v)$ which, of course, has only constant roots, it follows that in the terminology of [14; Section 5], each point of instability of $P(v)-t$ is "simple". It then follows from [14; Lemma 24] that if a factor $v-u_{j}(t)$ is removed from the right side of (27), the remaining polynomial does not have $M_{j}$ for a point of instability, and hence $M_{k} \neq M_{j}$ if $k \neq j$. Thus the set $\left\{\boldsymbol{M}_{0}, \ldots, M_{n-1}\right\}$ has precisely $n$ elements, and thus coincides with the set $\left\{\sigma_{j} \sigma t^{1 / n}: j=0, \ldots, n-1\right\}$. Hence, by renumbering if necessary, we can assume in (27) that

$$
u_{j} \sim \sigma_{j} \sigma t^{1 / n} \quad \text { over } \quad F(-\pi, \pi) \quad \text { for } j=0, \ldots, n-1,
$$

where $\sigma_{j}$ and $\sigma$ are given in (28).

We now let $w(z) \not \equiv 0$ be any solution of (1). Of course $w(z)$ is an entire function, and we set,

$$
W(t)=w\left(u_{0}(t)\right),
$$

so that $W(t)$ is admissible in $F(-\pi, \pi)$. Differentiating (30) twice, and substituting into (1), we find that $W(t)$ solves the equation,

$$
u_{0}^{\prime}(t) W^{\prime \prime}-u_{0}^{\prime \prime}(t) W^{\prime}+\left(u_{0}^{\prime}(t)\right)^{3} A\left(u_{0}(t)\right) W=0 .
$$


Since $u_{0}(t)$ and $W(t)$ are admissible in $F(-\pi, \pi)$, and since $u_{0}^{\prime} \sim(\sigma / n) t^{(1 / n)-1}$ over $F(-\pi, \pi)$ from $(29)$ and $\S 2(b)$, it follows from Lemma A that for some $K_{1}>0$, both $u_{0}(t)$ and $W(t)$ are analytic, and $u_{0}^{\prime}(t)$ is nowhere zero, on the set,

$$
|\operatorname{Arg} t|<\pi / 2, \quad|t| \geqslant K_{1} .
$$

Now let $\alpha$ be defined by (16), and let $p$ be any integer. Clearly, the set (32) contains the semi-infinite strip $D_{p}$ defined by,

$$
\alpha+2 \pi p-\pi<\operatorname{Im} t<\alpha+2 \pi p+\pi, \quad \operatorname{Re} t \geqslant K_{1},
$$

and so $u_{0}(t)$ and $W(t)$ are analytic, and $u_{0}^{\prime}$ has no zeros, on $D_{p}$. We now set,

$$
L(\zeta)=\log \zeta+i(\alpha+2 \pi p) \quad \text { for }|\operatorname{Arg} \zeta|<\pi .
$$

Then clearly, on the set,

$$
|\operatorname{Arg} \zeta|<\pi, \quad|\zeta| \geqslant \exp \left(K_{1}\right)
$$

the values of $t=L(\zeta)$ lie in $D_{p}$, and so the functions,

$$
U_{0}(\zeta)=u_{0}(L(\zeta)) \quad \text { and } \quad v_{0}(\zeta)=W(L(\zeta))
$$

are both analytic, and $U_{0}^{\prime}$ has no zeros, on the set (35). Differentiating each of the functions $U_{0}$ and $v_{0}$ twice, and using (31), it follows that $v_{0}(\zeta)$ satisfies the differential əquation,

$$
U_{0}^{\prime}(\zeta) v^{\prime \prime}-U_{0}^{\prime \prime}(\zeta) v^{\prime}+\left(U_{o}^{\prime}(\zeta)\right)^{3} A\left(U_{0}(\zeta)\right) v=0
$$

We now will require certain facts about $U_{0}(\zeta)$. We prove:

LEMma D. - (a) The following four asymptotic relations hold over $F(-\pi, \pi)$ :

$$
\begin{aligned}
& U_{0} \sim \sigma(\log \zeta)^{1 / n}, \quad U_{0}^{\prime} \sim(\sigma / n) \zeta^{-1}(\log \zeta)^{(1 / n)-1}, \\
& U_{0}^{\prime \prime} \sim-(\sigma / n) \zeta^{-2}(\log \zeta)^{(1 / n)-1} \\
& A\left(U_{0}(\zeta)\right) \sim d_{\beta} \sigma^{\beta} e^{i \alpha m} \zeta^{m}(\log \zeta)^{\beta / n}
\end{aligned}
$$

where $\beta, \boldsymbol{d}_{\beta}$, and $\alpha$ are defined in the statement of Theorem 1 , and $\sigma$ is given in (28).

(b) Let $\Gamma^{*}$ denote the field obtained by adjoining $U_{0}(\zeta)$ to the field of rational functions of $\zeta$. Then, $\Gamma^{*}$ has the following two properties: (i) $\Gamma^{*}$ contains the coefficients of $v^{\prime \prime}, v^{\prime}$, and $v$ in the equation (37); (ii) If $r \geqslant 1$ and $s \geqslant 1$, and if $N_{1}, \ldots, N_{s}$ are 

elements of $\Phi_{r}($ see $\S 2(c))$, while $f_{1}, \ldots, f_{s}$ are elements of $\Gamma^{*}$, then $\sum_{j=1}^{s} N_{j} f_{j}$ is either
identically zero or is $\sim$ to an element of $\Phi_{r}$ over $F(-\pi, \pi)$.

Proof. - Part $(a)$. In view of $(27)$ and the definition $(36)$ of $U_{0}(\zeta)$, clearly $U_{0}(\zeta)$ is a root of the polynomial in $v$ defined by,

$$
P(v)-L(\zeta)
$$

Now, for any $r \geqslant 1$, the coefficients of this polynomial lie in the logarithmic field $\Psi_{r}$ (see $\S 2(c)$ ), and thus by Lemma $\mathrm{C}$, there exists a logarithmic field $\Gamma_{r}$ of rank $r$ over $F(-\pi, \pi)$ such that,

$$
U_{0} \text { belongs to } \Gamma_{r} \text {. }
$$

Since $U_{0}$ belongs to $\Gamma_{1}$, there is a logarithmic monomial $N$ of rank $\leqslant 1$ such that $U_{0} \sim N$ over $F(-\pi, \pi)$. Hence from [2; Lemma $\left.5(c)\right]$, the monomial $N$ must be a point of instability (see [14; Section 5]) of (41). The algorithm in [14; Lemma 28] then shows that $N$ must be of the form $\sigma_{j} \sigma(\log \zeta)^{1 / n}$ for some $j=0,1, \ldots, n-1$ where $\sigma_{j}$ and $\sigma$ are as in (28). Hence for some $j=0,1, \ldots, n-1$,

$$
\bar{U}_{\mathbf{0}}(\zeta) \sim \sigma_{j} \sigma(\log \zeta)^{1 / n} \quad \text { over } F(-\pi, \pi) .
$$

We will prove that $\sigma_{j}=1$ in (43) which will prove the first relation in (38). To this end, we note that if $x$ denotes a sufficiently large real variable, then by (43), we have

$$
U_{0}(x) / \sigma_{j} \sigma(\ln x)^{1 / n} \rightarrow 1 \quad \text { as } x \rightarrow+\infty \text {. }
$$

Writing $x=e^{s}$ where $s$ is real, we obtain,

$$
u_{0}(s+i \lambda) / \sigma_{j} \sigma s^{1 / n} \rightarrow 1 \text { as } s \rightarrow+\infty,
$$

where $\lambda=\alpha+2 \pi p$ from (34) and (36). However, from (29), noting that $\sigma_{0}=1$. and that by Lemma A, the elements of $F(-\pi, \pi)$ contain the points $s+i \lambda$ for all sufficiently large $s$, it follows that,

$$
u_{0}(s+i \lambda) / \sigma(s+i \lambda)^{1 / n} \rightarrow 1 \quad \text { as } s \rightarrow+\infty .
$$

Thus from (45) and (46), we obtain,

$$
(s+i \lambda)^{1 / n} / \sigma_{j} s^{1 / n} \rightarrow 1 \quad \text { as } s \rightarrow+\infty .
$$

Noting that $\operatorname{Arg}(s+i \lambda) \rightarrow 0$ as $s \rightarrow+\infty$, it now follows from (47) that $\sigma_{j}=1$ proving the first relation in (38). The second relation in (38), and the relation (39) now both follow from $\S 2(b)$. 
To prove $(40)$, we note that since $U_{0}(\zeta)$ is a root of $(41)$, we have

$$
A\left(U_{0}(\zeta)\right)=\sum_{j=\alpha}^{m} Q_{j}\left(U_{0}(\zeta)\right) e^{i j \alpha} \zeta^{j} .
$$

Since each $Q_{j}(z)$ is a polynomial in $z$, the relation $(40)$ now follows immediately from (38) and the representation for $Q_{m}(z)$ in the statement of the theorem. This proves Part $(a)$.

To prove Part $(b)$, we observe first that since $D_{0}(\zeta)$ is a root of $(41)$, we have by differentiation that,

$$
U_{0}^{\prime}=\left(\zeta P^{\prime}\left(U_{0}(\zeta)\right)\right)^{-1}
$$

so that $U_{0}^{\prime}$ belongs to $I^{*}$. Differentiating again shows that $U_{0}^{\prime l}$ belongs to $T^{*}$. The representation (48) shows that $A\left(U_{0}(\zeta)\right)$ also belongs to $\Gamma^{*}$ proving (i) of Part $(b)$. To prove (ii), we observe that by (42), it follows that for each $r \geqslant 1$, the field $\Gamma^{*}$ is contained in $\Gamma_{r}$. Thus the linear combination $\sum_{j=1}^{s} N_{j} f_{j}$ described in (ii) also belongs to $\Gamma_{r}$, and the conclusion now follows from the fact that $\Gamma_{r}$ is a logarithmic field of rank $r$ over $F(-\pi, \pi)$. Thus Lemma $D$ is proved.

We now consider the function $v_{0}(\zeta)$ defined in (36) from an arbitrary solution $w(z) \neq 0$ of $(1)$. We prove:

LEMLMA E. - Let $\theta_{0}, \ldots, \theta_{m}$ be defined as in (17). Then, for each $j=0,1, \ldots, m-1$, there exists an element $V_{j}$ of $F\left(\theta_{j}, \theta_{j+1}\right)$ on which $v_{0}(\zeta)$ has no zeros.

Proof. - We consider the Riccati equation obtained from (37) by the substitution $u=v^{\prime} / v$ namely,

$$
U_{0}^{\prime}(\zeta)\left(u^{\prime}+u^{2}\right)-U_{0}^{\prime \prime}(\zeta) u+\left(U_{0}^{\prime}(\zeta)\right)^{3} A\left(U_{0}(\zeta)\right)=0
$$

We want to apply [12; Theorem III, p. 72] to this equation, and so we must verify the hypotheses of this theorem for equation (50). First, the coefficients of (50) do lie in a "logarithmic domain over $F(-\pi, \pi)$ " as defined in [12; Section 49]. The conclusion of Lemma D, Part $(b)$ (together with the fact that $T^{*}$ is a field), shows that $\Gamma^{*}$ is indeed a logarithmic domain of rank $\leqslant 1$ over $F(-\pi, \pi)$, and contains the coefficients of (50). We now must compute the principal monomials of (50). Using the algorithm in [12; Theorem, I, p. 28], the asymptotic relations (38), (39), (40), show that (50) possesses two principal monomials, $N_{1}$ and $N_{2}$, given by

$$
\pm \pi \zeta^{(m-2) / 2}(\log \zeta)^{(\beta+2-2 n) / 2 n}
$$

where $K$ is a root of the equation,

$$
\boldsymbol{K}^{2}+\sigma^{\beta+2} a_{\beta} e^{i \alpha m} n^{-2}=0 .
$$


The condition that these monomials be "simple (in the sense of [12; Section $68(f)]$ ) is satisfied since both roots of (52) are simple. The condition that the equation (50) be "asymptotically non-singular " at both $N_{1}$ and $N_{2}$ (in the sense of [12; Section 77, p. 36]) is easily seen to be satisfied since the partial derivative with respect to $u^{\prime}$ of the left side of (50) is just the function $U_{0}^{\prime}(\zeta)$. Finally, we must compute the "type" (as defined in [12; Section 82]) for each of the monomials $N_{1}$ and $N_{2}$. Using the formula for the type given in [12; Section. 81], and the asymptotic relations (38) and (39), we find that the type $T_{j}$ of $N_{j}$ is simply $2 N_{j}$ for $j=1,2$. Thus, to apply [12; Theorem III], we must consider the two functions,

$$
\operatorname{Cos}((m / 2) \theta+\arg ( \pm 2 K)) \quad \text { on }(-\pi, \pi) .
$$

(Of course, each of these functions is the negative of the other.) Using (52) and (28), we find that for one of the values of $K$, we have

$$
\arg (2 K)=((\beta+2) \pi / 4 n)+\left(\left(\arg d_{\beta}\right) / 2\right)+(\alpha m) / 2+\pi / 2 .
$$

Hence from the definition of $\alpha$ in (16), we find that $\arg (2 K)=-(m \pi / 2)+\pi / 2$, and so the functions in (53) are just $\pm \operatorname{Sin}((m / 2)(\theta-\pi))$, whose zeros on $[-\pi, \pi]$ are precisely at the points $\theta=\theta_{0}, \ldots, \theta_{m}$ given in (17). Thus from [12; Theorem III (b)] applied to each of $N_{1}$ and $N_{2}$, we can assert that for each $j=0,1, \ldots$, $\ldots, m-1$, the equation (50) possesses two solutions $u_{1 j}$ and $u_{2 j}$, admissible in $F\left(\theta_{j}, \theta_{j+1}\right)$, and such that,

$$
u_{1 j} \sim N_{1} \text { and } u_{2 j} \sim N_{2} \text { over } F^{\prime}\left(\theta_{j}, \theta_{j_{+1}}\right) .
$$

Clearly, if we take any functions $v_{1 j}$ and $v_{2^{j}}$ in $F\left(\theta_{j}, \theta_{j+1}\right)$ of the forms $v_{1 j}=\exp \int u_{1 j}$ and $v_{2 j}=\exp \int u_{2 j}$, then these functions are solutions of (37). Noting that $2 K$ and $K$ have the same arguments, it is clear from Lemma B, Part $(c)$ that the functions in (53) are simply the indicial functions $I\left(N_{1}, \theta\right)$ and $I\left(N_{2}, \theta\right)$ defined in Lemma $\mathrm{B}$, and since one is the negative of the other, it follows from Lemma $\mathrm{B}$, Part $(c)$, that for each $j$, the ratio $v_{1 j} / v_{2 j}$ either tends to zero or infinity in $F\left(\theta_{j}, \theta_{j+1}\right)$. It follows that $v_{1 j}$ and $v_{2 j}$ are linearly independent solutions of (37). Now, the function $v_{0}(\zeta)$ is admissible in each $F\left(\theta_{j}, \theta_{j+1}\right)$ since it is analytic on the set (35), and we know $v_{0}(\zeta)$ also satisfies (37). Noting that (37) has no singular points on the set (35) (since $U_{0}^{\prime}$ has no zero on (35)), it follows that for each $j$, there is an element of $F\left(\theta_{j}, \theta_{j+1}\right)$ on which we can write $v_{0}=c_{1 j} v_{1 j}+c_{2 j} v_{2 j}$ for some constants $e_{1 j}, c_{2 j}$, not both zero. If one of these constants is zero, then $v_{0}$ is a multiple of either $v_{1 j}$ or $v_{2 j}$, and it is clear from the form of these solution that they are nowhere zero on some element of $F\left(\theta_{j}, \theta_{j+1}\right)$. If both constants $c_{1 j}$ and $c_{2 j}$ are nonzero, then noting that a zero of $v_{0}$ can occur only where $v_{1 j} / v_{2 j}=-c_{2 j} / c_{1 j}$, we see that since the ratio $v_{1 j} / v_{2 j}$ either tends to zero or to infinity in $F\left(\theta_{j}, \theta_{i+1}\right)$, there is an element of $F\left(\theta_{j}, \theta_{j+1}\right)$ on which $v_{0}(\zeta)$ has no zeros. This proves Lemma $\mathrm{E}$. 
We now let $\delta>0$ be arbitrary. Then by Lemma A (and Lemma $\mathbf{E}$ ), for each $j=0,1, \ldots, m-1$, there exists $R_{\delta i}>0$ such that $v_{0}(\zeta)$ has no zeros on the set

$$
\theta_{j}+\delta \leqslant \operatorname{Arg} \zeta \leqslant \theta_{j+1}-\delta, \quad|\zeta| \geqslant R_{\delta j} .
$$

(Of course, since $v_{0}(\zeta)$ also depends on the integer $p$ in (34), we will write $R_{\delta j}=R_{\delta j p}$ ) Now, let $D_{\delta j p}$ denote the set,

$$
\theta_{j}+\alpha+2 \pi p+\delta<\operatorname{Im} t<\theta_{j+1}+\alpha+2 \pi p-\delta, \quad \operatorname{Re} t \geqslant \log R_{\delta j p} .
$$

Then, if $t$ belongs to $D_{\delta j p}$, clearly the point $\zeta=e^{t-i(\alpha+2 \pi p)}$ lies in the set (56), and $t=L(\zeta)$ where $L(\zeta)$ is given by $(34)$. Since $v_{0}(\zeta)=W(t)$ by $(36)$, and since $v_{0}(\zeta)$ is nowhere zero on $(56)$, it follows that,

$$
W(t) \text { has no zeros on } D_{\delta j p}{ }^{4} \text {. }
$$

To obtain a corresponding region for the original solution $w(z)$, we need the following result (where we are using the notation established in (14), (27), (28) and $(29))$ :

Lewrar F. - Define functions $h_{0}(t), \ldots, h_{n-1}(t)$ by the formula,

$$
u_{j}(t)=\sigma_{j} \sigma^{1 / n}\left(1+h_{j}(t)\right)
$$

and let $\varepsilon$ be any number such that $0<\varepsilon<\pi / n$. Then there exist constants $K_{1 \varepsilon}>0$ and $K_{2 \varepsilon}>0$ such that the following two conclusions hold:

(A) For each $j=0,1, \ldots, n-1$, the functions $u_{j}(t)$ and $h_{j}(t)$ are analytic on the set,

$$
S_{1 \varepsilon}:(-\pi / 2)+(n \varepsilon / 2) \leqslant \Delta \operatorname{Arg} t \leqslant(\pi / 2)-(n \varepsilon / 2), \quad|t| \geqslant K_{1 \varepsilon}
$$

and we have,

$$
\left|\operatorname{Arg}\left(1+h_{j}(t)\right)\right|<\varepsilon / 2 \quad \text { on } \quad S_{1 \varepsilon}^{1} \quad \text { for each } j \text {. }
$$

(B) Let $T_{18}$ denote the set,

$$
T_{1 \varepsilon}: \varepsilon \leqslant \operatorname{Arg} z \leqslant(\pi / n)-\varepsilon, \quad|z| \geqslant K_{2 \varepsilon} .
$$

Then, for $z$ in $T_{1 \varepsilon}$ we have that the point $t=P(z)$ belongs to $S_{1 \varepsilon}$ and $z=u_{0}(P(z))$.

Proof. - Part $(A)$. Clearly there exists a number $\varepsilon_{1}$ with $0<\varepsilon_{1}<1$, such that on the disk $|z-1|<\varepsilon_{1}$, we have $|\operatorname{Arg} z|<\varepsilon / 2$. Now $u_{j}$ and $h_{j}$ are clearly admissible 
in $F(-\pi, \pi)$ and by $(29)$, we have $h_{j} \rightarrow 0$ in $F(-\pi, \pi)$. Thus there exists an element $S$ in $F(-\pi, \pi)$ on which $u_{j}$ and $h_{j}$ are analytic, and $\left|h_{j}(t)\right|<\varepsilon_{i}$. Then on $S$, clearly $1+h_{j}(t)$ lies in the disk $|z-1|<\varepsilon_{1}$, and so (61) holds on $S$. By Lemma $A$, clearly $S$ contains a set of the form $S_{1 \varepsilon}$ for some $K_{1 \varepsilon}>0$ which proves Part (A).

Part (B): From (14), we can write,

$$
P(z)=i b z^{n}(1+h(z)), \quad \text { where } h \rightarrow 0 \text { as } z \rightarrow \infty .
$$

Letting $\varepsilon_{1}$ be as in Part (A), there exists $K_{3 \varepsilon}>0$ such that $|h(z)|<\varepsilon_{1}$ for $|z| \geqslant K_{3 \varepsilon}$, and hence by the property of $\varepsilon_{1}$, we have,

$$
|\operatorname{Arg}(1+h(z))|<\varepsilon / 2 \leqslant n \varepsilon / 2 \text { for }|z| \geqslant K_{3 \varepsilon} .
$$

Now consider the set,

$$
T_{3 \varepsilon}: \varepsilon \leqslant \operatorname{Arg} z \leqslant(\pi / n)-\varepsilon, \quad|z| \geqslant K_{3 \varepsilon} .
$$

If $z=r e^{i \theta}$ belongs to $T_{3 \varepsilon}$, with $\varepsilon \leqslant \theta \leqslant(\pi / n)-\varepsilon$, then noting that $b<0$ in (63), it follows from (64) that we can write,

$$
P\left(r e^{i \theta}\right)=e^{-i \pi / 2}|b| r^{n} e^{i n \theta}|1+h(z)| e^{i \varphi(z)}
$$

where $|\varphi(z)| \leqslant n \varepsilon / 2$. It then follows that,

$$
-(\pi / 2)+n \varepsilon / 2 \leqslant \operatorname{Arg} P\left(r e^{i \theta}\right) \leqslant(\pi / 2)-n \varepsilon / 2 .
$$

Also,

$$
\left|P\left(r e^{i \theta}\right)\right| \geqslant|b| r^{n}\left(1-\varepsilon_{1}\right),
$$

and so if we choose $K_{2 \varepsilon}>0$ so large that for $r \geqslant K_{2 \varepsilon}$, the right side of (68) exceeds $K_{1 \varepsilon}$, then for $z$ in $T_{1 \varepsilon}$, we see from (67) that $P(z)$ belongs to the set $S_{1 \varepsilon}$ in (60). Finally, we must prove $z=u_{0}(P(z))$ for $z$ in $T_{1 \varepsilon}$. Let $z$ belong to $T_{1 \varepsilon}$, and let $z_{1}=u_{0}(P(z))$. Now the constant function $v(t) \equiv z_{1}$ is certainly admissible in $F(-\pi, \pi)$, and for the value $t=P(z)$, we clearly have $v(t)-u_{0}(t)=0$ by definition of $z_{1}$. Thus the right side of (27) is zero for this choice of $v$ and value of $t$. Thus the left side of (27) must also be zero, which yields,

$$
P\left(z_{1}\right)=P(z)
$$

Since $P(z)$ belongs to $S_{1 \varepsilon}$ by the first part of this proof, we also have,

$$
P\left(z_{1}\right) \text { belongs to } S_{1 \varepsilon} \text {. }
$$


The constant function $v(t) \equiv z$ is admissible in $F(-\pi, \pi)$, and $(69)$ shows that the left side of $(27)$ is zero for $v=v(t)$ at the value of $t=P\left(z_{1}\right)$. Hence, some factor on the right side of (27) must be zero for this choice of $v(t)$ and $t$, and thus for some $j$ in $\{0,1, \ldots, n-1\}$, we have $z=u_{j}\left(P\left(z_{1}\right)\right)$. In view of $(69)$, we thus obtain,

$$
z=u_{j}(P(z)) \text {. }
$$

The proof of Part $(b)$ will be complete if we can show $j=0$. To this end, we know from the first part of the proof that $P(z)$ belongs to $S_{1 \varepsilon}$. Hence we can write $P(z)=$ $=\boldsymbol{R} e^{i \varphi}$, where,

$$
R \geqslant K_{1 \varepsilon} \quad \text { and } \quad(-\pi / 2)+(n \varepsilon / 2) \leqslant \varphi \leqslant(\pi / 2)-(n \varepsilon / 2) .
$$

In view of (59), (and the definitions of $\sigma$ and $\sigma_{j}$ given in (28)), we thus have,

$$
u_{j}(P(z))=|b|^{-1 / n} R^{1 / n}\left|1+h_{j}(P(z))\right| e^{i \varphi_{0}},
$$

where,

$$
\varphi_{0}=(2 \pi j / n)+(\pi / 2 n)+(\varphi / n)+\operatorname{Arg}\left(1+h_{j}(P(z))\right) .
$$

In view of $(61)$ and $(72)$, we see that,

$$
(2 \pi j / n) \leqslant \varphi_{0} \leqslant(2 \pi j / n)+(\pi / n) .
$$

On the other hand, since $z$ belongs to $T_{1 \varepsilon}$, we can write (by (62)), $z=R_{1} e^{i \varphi_{1}}$, where,

$$
R_{1} \geqslant K_{2 \varepsilon} \quad \text { and } \quad \varepsilon \leqslant \varphi_{1} \leqslant(\pi / n)-\varepsilon .
$$

In view of (71) and (73), it follows that $\varphi_{0}-\varphi_{1}=2 \pi d$ for some integer $d$. From (75) and (76), we thus see that,

$$
(\pi / n)(2 j-1)+\varepsilon \leqslant 2 \pi d \leqslant(\pi / n)(2 j+1)-\varepsilon .
$$

Multiplying the inequality (77) by $n / 2 \pi$, we find that,

$$
-\frac{1}{2}+\varepsilon n / 2 \pi \leqslant n d-j \leqslant \frac{1}{2}-(\varepsilon n / 2 \pi) .
$$

Since $n d-j$ is an integer, we thus obtain $n d-j=0$. Hence $d=j / n$, and since $0 \leqslant j \leqslant n-1$, while $d$ is an integer, we must have $d=0$ and $j=0$, which completes the proof of Part (B).

We are now ready to complete the proof of Theorem 1. Let $\varepsilon>0$ and $\delta>0$ be arbitrary, and let $p$ and $j$ be integers, with $0 \leqslant j \leqslant m-1$. Let the set $A_{\varepsilon \delta p j}$ be 
as defined in the statement. (We can assume that $\varepsilon<\pi / n$, for otherwise the set $A_{\varepsilon \delta p j}$ is empty.) Let $z$ be any point in $A_{\varepsilon \delta p j}$ satisfying the additional conditions that (see (57) and (62)),

$$
|z| \geqslant K_{2 \varepsilon} \quad \text { and } \quad \operatorname{Re} P(z) \geqslant \log R_{\delta j p}
$$

In view of Lemma $F$, we have,

$$
P(z) \quad \text { belongs to } S_{1 \mathrm{~s}} \text { and } z=u_{0}(P(z)),
$$

and, in addition, by (18) and (57), we have that

$$
P(\approx) \quad \text { belongs to } D_{\delta j p} \text {. }
$$

But by (30) and (80), we have $w(z)=W(P(z))$, and so by (58) and (81), we see that $w(z) \neq 0$. Hence $w(z)$ has no zeros at those points in $A_{\varepsilon \delta p j}$ which satisfy (79). To conclude the proof, we must show that $w(z)$ can have at most finitely many zeros in each of the sets,

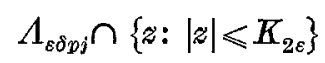

and

$$
A_{\varepsilon \delta \phi j} \cap\left\{z: \operatorname{Re} P(z) \leqslant \log R_{\delta j p}\right\} \cap\left\{z:|z| \geqslant K_{2 \varepsilon}\right\}
$$

Of course, being an entire function, not identically zero, $w(z)$ can have only finitely many zeros in the bounded set (82). For points $z$ in the set (83), we have by Lemma $F$ that $P(z)$ belongs to $S_{1 \varepsilon}$ which (see (60)) implies that $P(z)$ lies in the right half-plane. Thus, the set (83) is certainly contained in the set

$$
\Lambda_{\varepsilon \delta p j} \cap\left\{z: 0 \leqslant \operatorname{Re} P(z) \leqslant \log R_{\delta j p}\right\} .
$$

But from (18), it follows that for all points $z$ in the set (84), both $\operatorname{Re} P(z)$ and $\operatorname{Im} P(z)$ are bounded. Thus $P(z)$ is bounded on the set (84), from which it follows that (84) is a bounded set, and so $w(z)$ can have only finitely many zeros on it. This concludes the proof of Theorem 1.

4. - Definition $[12 ; \S 14]$. - An admissible function $f(z)$ in $F(a, b)$ is called trivial in $F(a, b)$ if for every positive integer $p$, we have $f \ll z^{-p}$ in $F(a, b)$.

5. - Proof of Theorem 2. - We consider the Riccati equation obtained by setting $u=v^{\prime} / v$ in (1), namely,

$$
u^{\prime}+u^{2}+A(z)=0 .
$$


Since $b>0$ in (14), it follows from Lemma B, Part (c), that,

$$
e^{P(z)} \text { is trivial in } F(0, \pi / n) .
$$

We will first prove Part (II) of the theorem, namely the case where $q=0$. From (2) and (86), we see that in $F(0, \pi / n)$,

$$
A(z)=Q_{0}(z)+T_{1}(z) \quad \text { and } \quad Q_{0} \sim e_{2} z^{\lambda},
$$

where $T_{1}$ is trivial in $F^{\prime}(0, \pi / n)$. We want to apply [12; Theorem III] to equation (85). We first note that the coefficients of (85) belong to a "logarithmic domain of rank zero over $F(0, \pi / n)$ " (as defined in $[12 ; \S 49]$ ) since by $[12 ; \S \S 52,53(c)$, $128(e)]$, such a set is formed by all functions of the form $f+T$, where $f$ belongs to $\Psi_{0}$ (see $\S 2(e)$ ) and $T$ is trivial in $F(0, \pi / n)$, and, by (87), $A(z)$ has this form. Using the algorithm in $[12 ; \S 28]$, we find that $(85)$ possesses two principal monomials $M_{1}$ and $M_{2}$ given by

$$
\pm K z^{\lambda / 2}, \quad \text { where } \quad K^{2}=-c_{\lambda} \text {. }
$$

Both are simple, and the condition of asymptotic non-singularity is immediately verified. The type for $M_{j}$ is $2 M_{i}$, and thus we must consider the functions,

$$
\operatorname{Cos}(((\lambda+2) \theta / 2)+\arg ( \pm 2 K)) \text { on }(0, \pi / n) .
$$

Of course the two functions in (89) are just $\pm H(\theta)$ where $H(\theta)$ is given by (19). If the numbers $\psi_{0}, \ldots, \psi_{N_{+}+1}$ are defined as in the statement of Part (II) of Theorem 2, then by [12; Theorem III], it follows that for each $j=0,1, \ldots, N$, the equation (85) possesses two solutions $u_{1 j}$ and $u_{2 j}$ admissible in $F\left(\psi_{j}, \psi_{i_{+1}}\right)$ such that

$$
u_{1 j} \sim M_{1} \quad \text { and } \quad u_{2 j} \sim M_{2} \quad \text { over } \quad F\left(\psi_{j}, \psi_{j+1}\right) .
$$

Letting $v_{1 j}=\exp \int u_{1 j}$ and $v_{2}=\exp \int u_{2 j}$, it follows that $v_{1 j}$ and $v_{2 j}$ solve (1), and since the functions (89) are the indicial functions (see $\S 2(b)$ ) of $M_{1}$ and $M_{2}$, and each is the negative of the other, it follows from Lemma B, Part $(c)$ that in $F\left(\psi_{j}, \psi_{j+1}\right)$, the ratio $v_{1 j} / v_{2 j}$ either tends to zero or infinity. Since any solution $w(z) \neq 0$ of $(1)$ must be a linear combination of $v_{1 j}$ and $v_{2 j}$ in $F\left(\psi_{j}, \psi_{j+1}\right)$, it thus follows that for each $j$, there is an element of $F\left(\psi_{j}, \psi_{i_{+1}}\right)$ on which $w(z)$ has no zeros. The conclusion now follows from Lemma $\mathrm{A}$ and the fact that the entire function $w(z)$ can have at most finitely many zeros in any bounded set. This proves Part (II).

In Part (I), we have $q>0$ in (2), and from (86), we can write,

$$
A=e^{q P}\left(Q_{q}+T_{2}\right) \quad \text { where } T_{2} \text { is trivial in } \quad F(0, \pi / n) .
$$


Introducing a new unknown function $U$ in (85) by $U=u e^{-a P}$, we find that $U$ satisfies the equation,

$$
U^{\prime}+q^{\prime} P^{\prime} U+e^{Q P} U^{2}+Q_{a}+T_{2}=0
$$

This equation has coefficients in the same logarithmic domain as appeared in the proof of Part (II) (since $e^{q P}$ is trivial). It is easy to see from [12; § 28], that (92) possesses only one principal monomial $M$, namely,

$$
M=-\left(d_{\sigma} /(i q n b)\right) z^{\sigma+1-n},
$$

where $d_{\sigma} z^{\sigma}$ is the leading term of $Q_{q}$. The conditions of being simple and asymptotically non-singular are easily verified, and the type is found to be $i q n b z^{n-1}$. Since the function $\cos (n \theta+\arg (i q n b))$ has no zeros on $(0, \pi / n)$, it follows from $[12$; Theorem III] that in $F(0, \pi / n)$, the equation (92) possesses a solution $U_{1}$ such that,

$$
U_{1} \sim M \quad \text { in } \quad F(0, \pi / n) .
$$

Hence $u_{1}=e^{a P} U_{1}$ solves (85), and thus,

$$
w_{1}=\exp \int e^{a P} U_{1} \quad \text { solves }(1)
$$

To find a second solution of (1), we introduce a new unknown function $W$ in (85) by

$$
W=e^{-\alpha P}\left(u-z^{-1}\right) .
$$

In view of (91), we find that $W$ solves the equation,

$$
W^{\prime}+\left(q P^{\prime}+2 z^{-1}\right) W+e^{q P} W^{2}+Q_{q}+T_{2}=0 .
$$

This equation has coefficients in the same logarithmic domain as in Part (II), and we again find that (97) has only one principal monomial, namely $M$ given by (93), and that the conditions of being simple and asymptotically non-singular are met. The type is again $i q n b z^{n-1}$, and as before, we can assert by [12; Theorem $\amalg I I$, that (97) possesses a solution $W_{1}$ such that,

$$
W_{1} \sim M \quad \text { in } \quad F(0, \pi / n) .
$$

Hence (85) possesses the solution,

$$
u_{2}=z^{-1}+e^{\alpha P} W_{1},
$$


and thus,

$$
w_{2}=\exp \int\left(z^{-1}+e^{q P} W_{1}\right)
$$

solves (1) in $F(0, \pi / n)$. The ratio $w_{2} / w_{1}$ of the two solutions has the form,

$$
w_{2} / w_{1}=z\left(\exp \int e^{q P}\left(W_{1}-\nabla_{1}\right)\right) .
$$

Since $e^{Q P}$ is trivial in $F(0, \pi / n)$ by (86), while $W_{1}-U_{1} \ll M$ by (94) and (98), it follows from Lemma B, Part $(b)$ that $w_{2} / w_{1}$ tends to infinity in $F(0, \pi / n)$. As before, it follows that for any solution $w(z) \neq 0$ of (1), there is an element of $F(0, \pi / n)$ on which $w(z)$ has no zeros. The conclusion of Part (I) now follows from Lemma A and the fact that $w(z)$ is entire.

6. - Proof of Theorem 3. - Part $(A)$. We are assuming the hypothesis and notation of Theorem 1 , and we let $a_{j p}, \varepsilon_{1}$ and $\delta_{1}$ be as in the statement. Now if $w(z) \neq 0$ is a solution of $(1)$, and we define $W(t)$ by $(30)$, then $W$ is analytic on the set (32) for some $K_{1}>0$, and satisfies equation (31). We now set,

$$
L_{1}(\zeta)=\log \zeta+i a_{j p},
$$

so that $L_{1}$ is analytic on the set (35), and the values $t=L_{1}(\zeta)$ lie in,

$$
a_{j p}-\pi<\operatorname{Im} t<a_{j p}+\pi, \quad \operatorname{Re} t \geqslant K_{1} .
$$

Thus, on the set (35), the functions

$$
U_{1}(\zeta)=u_{0}\left(L_{1}(\zeta)\right) \quad \text { and } \quad v_{1}(\zeta)=W\left(L_{1}(\zeta)\right)
$$

are both analytic. It is easy to eheck that $v_{1}(\zeta)$ solves (37) where $U_{0}$ is replaced by $U_{1}$, and hence $u_{1}=v_{1}^{\prime} / v_{1}$ solves the equation (which we will denote by $\left(50^{\prime}\right)$ ) which is the equation obtained from (50) by replacing $U_{0}$ by $U_{1}$.

Now, from (27), clearly $U_{1}(\zeta)$ is a root of the polynomial $P(v)-L_{1}(\zeta)$, and it follows exactly as in the proof of Lemma $D$, that the asymptotic relations (38) and (39) for $U_{0}, U_{0}^{\prime}$ and $U_{0}^{\prime \prime}$ are valid for $U_{1}, U_{1}^{\prime}$ and $U_{1}^{\prime \prime}$ respectively, and that Part $(b)$ of Lemma $\mathrm{D}$ holds for $U_{1}$ in place of $U_{0}$. Of course, (40) does not hold for $U_{1}$ and must be replaced by,

$$
A\left(U_{1}(\zeta)\right) \sim d_{\beta} \sigma^{\beta} e^{i a_{j} m} \zeta^{m}(\log \zeta)^{\beta / n}
$$

since $e^{L_{1}(\zeta)}=\zeta e^{i a_{j} p}$. These asymptotic relations show that the principal monomials of $\left(50^{\prime}\right)$ are given by,

$$
N_{3}=K_{2} \zeta^{(m-2) / 2}(\log \zeta)^{(\beta+2-2 n) / 2 n}, \quad \text { and } \quad N_{4}=-N_{3},
$$


where $K_{2}$ is a root of

$$
K_{2}^{2}+\sigma^{\beta+2} d_{\beta} e^{i \alpha_{j p} m} n^{-2}=0 .
$$

As in the proof of Lemma $\mathrm{E}$, the type of $\left(50^{\prime}\right)$ at $N_{j}$ is $2 N_{j}$ for $j=3,4$, and so to apply [12; Theorem III], we must consider the two functions,

$$
\operatorname{Cos}\left((m / 2) \theta+\arg \left( \pm 2 K_{2}\right)\right)
$$

which are of course negatives of each other. From the definitions of $\sigma, d_{\beta}$, and $a_{j p}$ (see (16) and (28)), it is easy to see that the roots $\pm \boldsymbol{K}_{2}$ of (107) are purely imaginary, and we will designate by $K_{2}$, the one with the positive imaginary part, say,

$$
K_{2}=i c_{1} \quad \text { where } e_{1}>0 .
$$

Clearly, both functions in (108) vanish at $\theta=0$, and change sign through $\theta=0$. We can now apply [12; Theorem III, Part $(b)]$ in its full strength to assert that there exist $e_{2}>0$ with $c_{2}<\pi$, and solutions $u_{3}(\zeta)$ and $u_{4}(\zeta)$ of equation $\left(50^{\prime}\right)$ such that,

$$
u_{3} \sim N_{3} \quad \text { and } \quad u_{4} \sim N_{4} \quad \text { in } \quad F\left(-c_{2}, e_{2}\right)
$$

Choosing a convenient point $c>0$, and setting,

$$
v_{3}=\exp \int_{c}^{\zeta} u_{3} \quad \text { and } \quad v_{4}=\exp \int_{c}^{\zeta} u_{4}
$$

it is clear that $v_{3}$ and $v_{4}$ satisfy the equation (which we will call $\left(37^{\prime}\right)$ ) which is obtained from (37) by replacing $U_{0}$ by $U_{1}$. In view of Lemma $\mathrm{A}$, the domains of $v_{3}$ and $v_{4}$ include a set of the form,

$$
-c_{2} / 2<\operatorname{Arg} \zeta<c_{2} / 2, \quad|\xi| \geqslant K_{3},
$$

for some $K_{3}>0$. Thus, on the set,

$$
a_{j p}-\left(c_{2} / 2\right)<\operatorname{Im} t<a_{j_{p}}+\left(c_{2} / 2\right), \quad \operatorname{Re} t \geqslant \log K_{3},
$$

the functions $W_{k}(t)=v_{l k}\left(e^{i-i a_{j p}}\right)$ for $k=3,4$, are both analytic and satisfy (31).

Lemma G. - There exists a positive number $K_{4}>\log K_{3}$, such that for any $t$ satisfying

$$
a_{j p}-\left(c_{2} / 2\right)<\operatorname{Im} t<a_{j_{p}}+\left(c_{\mathrm{a}} / 2\right), \quad \operatorname{Re} t>K_{4},
$$


there exists a complex number $z$ satisfying the following five conditions:

$$
\begin{aligned}
& \varepsilon_{1}<\operatorname{Arg} z<(\pi / n)-\varepsilon_{1}, \\
& a_{j_{p}}-\left(c_{2} / 2\right)<\operatorname{Im} P(\approx)<a_{j_{p}}+\left(c_{2} / 2\right), \quad \operatorname{Re} P(z)>K_{4},
\end{aligned}
$$

and

$$
t=P(z) \quad \text { and } \quad z=u_{0}(t)
$$

Proof. - Let $\varepsilon>0$ be any number satisfying $\varepsilon<(\pi / 2 n)-\varepsilon_{1}$. For this $\varepsilon$, we apply Lemma $\mathrm{F}$ to obtain the constants $K_{1 \varepsilon}, K_{2 \varepsilon}$, and the sets $S_{1 \varepsilon}$ and $T_{1 \varepsilon}$, and we know (61) holds on $S_{1 \varepsilon}$ for $j=0$. We can obviously choose a constant $K_{4}>\log K_{3}$ so that the set (114) is contained in $S_{1 e}$. In addition, we can choose $K_{4}$ so large that on the set (114), we have $|\operatorname{Arg} t|<n \varepsilon / 2$. Then, for $t$ belonging to the set (114), we know that $t$ belongs to $S_{1 \varepsilon}$ so $z=u_{0}(t)$ exists. In addition, by (59) and (61) (for $j=0$, noting that $\sigma_{0}=1$ ) we obviously obtain,

$$
(\pi / 2 n)-\varepsilon<\operatorname{Arg} u_{0}(t)<(\pi / 2 n)+\varepsilon .
$$

By choice of $\varepsilon$, this shows $z$ satisfies (115). Since $z=u_{0}(t)$, we have $t=P(z)$, and so the remaining conditions (116) and (117) are satisfied, proving the lemma.

For convenience, we denote by $E$, the set of all complex numbers $z$ satisfying (115) and (116). (From Lemma G, it follows that $E$ is a nonempty open set.) Clearly if $z$ belongs to $E$, then $t=P(z)$ belongs to the set (113), and so the functions,

$$
w_{1}(z)=W_{3}(P(z)) \quad \text { and } \quad w_{2}(z)=W_{4}(P(z)),
$$

are defined and analytic on each component of $t$. Since $W_{3}(t)$ and $W_{4}(t)$ satisfy (31) on the set (114), and each element of the set (114) can be written as $P(z)$ for some $z$ in $E$ (by (117)), it easily follows that $w_{1}(z)$ and $w_{2}(z)$ are both solutions of equation (1) on the components of $E$. (Of course, they can be extended to entire functions.) We will prove that the solutions $w_{1}$ and $w_{2}$ satisfy the conclusion of Part (A). First, it is clear that $w_{1}$ and $w_{2}$ are linearly independent, for in the contrary case, it would follow that $W_{3}(t)$ and $W_{4}(t)$ are linearly dependent on the set (114) by Lemma G. However, this would imply the linear dependence of $v_{3}(\zeta)$ and $v_{4}(\zeta)$ on the set (112), which is false since by Lemma B, Part (c), and the fact that $\theta=0$ is an isolated zero of the functions (108), we see that the ratio $v_{3} / v_{4}$ either tends to zero or to infinity in $F\left(0, c_{2}\right)$.

We will conclude the proof of Part (A) by showing:

Lemra $H$. - Let $\delta_{1}>0$ be any number such that $\delta_{1}<\operatorname{Sin}\left(c_{2} / 2\right)$, and let $c_{3}$ and $c_{4}$ be nonzero complex numbers. Then the function $v(\zeta)=c_{3} v_{3}(\zeta)+c_{4} v_{4}(\zeta)$ possesses 
an infinite sequence $\zeta_{1}^{*}, \zeta_{2}^{*}, \ldots$ of zeros satisfying,

$$
\left|\zeta_{k}^{*}\right| \rightarrow+\infty \quad \text { as } \quad k \rightarrow \infty \text {, and } \quad\left|\operatorname{Arg} \zeta_{k}^{*}\right|<\delta_{1} \quad \text { for each } k \text {. }
$$

When Lemma $\mathrm{H}$ is proved, the proof of Part (A) will be completed as follows: The points $t_{k}=L_{1}\left(\zeta_{k}^{*}\right)$ will then be zeros of $e_{3} W_{3}(t)+c_{4} W_{4}(t)$ satisfying,

$$
a_{j_{p}}-\delta_{1}<\operatorname{Im} t_{k s}<a_{j_{p}}+\delta_{1}, \text { and } \operatorname{Re} t_{k} \rightarrow+\infty \text {. }
$$

By Lemma $\mathrm{G}$, if $k$ is sufficiently large, then $t_{k}=P\left(z_{k}\right)$ for some $z_{k}$ belonging to $E$, and clearly $z_{l}$ is a zero of $c_{3} w_{1}+c_{4} w_{2}$. These zeros will have infinite exponent of convergence because the sequence constructed in Lemma $\mathrm{H}$ will have strictly positive exponent by (129) below.

To prove Lemma $\mathrm{H}$, we set $s_{1}=-c_{4} / c_{3}$ and we rewrite the equation $v(\zeta)=0$ as,

$$
\exp \int_{c}^{\zeta}\left(u_{3}-u_{4}\right)=s_{1}
$$

In view of (106) and (110), we can write,

$$
u_{3}-u_{4}=2 N_{3}+E_{0}, \quad \text { where } E_{0} \ll N_{3} \text { in } F\left(-c_{2}, c_{2}\right) .
$$

We define,

$$
M_{3}(\zeta)=\left(4 K_{2} / m\right) \zeta^{m / 2}(\log \zeta)^{s} \quad \text { where } s=(\beta+2-2 n) / 2 n,
$$

so that by (106), we have,

$$
M_{3}^{\prime}=2 N_{3}+E_{1}, \quad \text { where } E_{1} \ll N_{3} \text { in } F(-\pi, \pi) .
$$

We now assert that we can write,

$$
\int_{G}^{\zeta}\left(u_{3}-u_{4}\right)=M_{3}+E_{2} \quad \text { where } E_{2} \ll M_{3} \text { in } F\left(-c_{2}, c_{2}\right) .
$$

To prove this, we consider the equation,

$$
y^{\prime}=u_{3}-u_{4}
$$

In view of (123) and (125), we see that under the change of dependent variable $y=M_{3}+M_{3} y_{1}$, the equation (127) becomes,

$$
\left(M_{3} / M_{3}^{\prime}\right) y_{1}^{\prime}+y_{1}=\left(E_{0}-E_{1}\right) /\left(2 N_{3}+E_{1}\right)
$$


The equation (128) is "normal over $F^{\prime}\left(-c_{2}, c_{2}\right)$ » in the sense of $[12 ; \S 83]$ with "divergence monomial" equal to $(m / 2) \xi^{-1}$, and hence by [12; Lemma 111], equation (128) possesses a solution $y_{1} \ll 1$ in $F\left(-c_{2}, c_{2}\right)$. Then, $y=M_{3}+M_{3} y_{1}$ solves (127), as does the left side of (126), and so they differ by a constant, which yields (126).

We now write $s_{1}=\left|s_{1}\right| e^{i \varphi}$ in (122), where $|\varphi| \leqslant \pi$, and in view of (126), the equation (122) is equivalent to,

$$
M_{3}(\zeta)+E_{2}(\zeta)=\log \left|s_{1}\right|+i(\varphi+2 \pi r), \quad \text { where } r=0, \pm 1, \ldots
$$

We will produce, for each sufficiently large integer $r$, a root $\zeta_{r}^{*}$ of (129) for which (120) holds, and thus Lemma $\mathbf{H}$ will be proved.

We set,

$$
G_{r}(\zeta)=M_{3}(\zeta)-2 \pi i r, \quad \text { for } r=0, \pm 1, \ldots
$$

and

$$
E_{3}(\zeta)=E_{\mathbf{2}}(\zeta)-\log \left|s_{\mathbf{1}}\right|-i \varphi
$$

so that by (126),

$$
E_{3} \ll M_{3} \quad \text { in } F\left(-c_{2}, c_{2}\right)
$$

and equation (129) becomes,

$$
G_{r}(\zeta)+E_{3}(\zeta)=0
$$

In view of (124) and (109), the equation $G_{r}(\zeta)=0$ is equivalent to,

$$
\left(4 c_{1} / m\right) \zeta^{m / 2}(\log \zeta)^{s}=2 \pi r
$$

and since $c_{\mathbf{1}}>0$ and $m>0$, it is clear that for each $r=1,2, \ldots$, there is a real number $\zeta_{r}$ in $(1,+\infty)$ for which,

$$
\dot{G}_{r}\left(\zeta_{r}\right)=0, \text { and }\left|\zeta_{r}\right| \rightarrow+\infty \text { as } r \rightarrow \infty .
$$

Now, with $\delta_{1}>0$ given as in Lemma $H$, we now choose $\delta_{2}>0$ satisfying the following conditions:

$$
\delta_{2}<\left(\operatorname{Sin} \delta_{1}\right) / 2 \quad \text { and } \quad \delta_{2}<\left|K_{2}\right| /\left(2 K_{5} K_{6} K_{7}\right)
$$


where

$$
\begin{aligned}
& K_{5}=4\left|K_{\mathrm{a}}\right|(|(m-2) / 2|+1), \\
& K_{6}=\frac{3}{2}(m / 2)-2+\frac{1}{2}(m / 2)-2, \\
& K_{7}=4^{s}+\frac{1}{2} s, \quad \text { where } s \text { is as in }(124) .
\end{aligned}
$$

Now from (125) and (106) (and (124)), we can write,

$$
M_{3}^{\prime \prime}=2 K_{2} \zeta^{(m / 2)-2}(\log \zeta)^{s}\left((m-2) / 2+s(\log \zeta)^{-1}\right)+E_{1}^{\prime} .
$$

Now $E_{1} \ll N_{3}$ in $F(-\pi, \pi)$, so by $\S 2(b)$ and (125), it follows that if $N_{3}$ is not a constant, then $M_{3}^{\prime \prime} \sim 2 N_{3}^{\prime}$ and $E_{1}^{\prime} \ll M_{3}^{\prime \prime}$. But if $N_{3}$ is a constant, we have $m=2$, $s=0$, and $E_{1}^{\prime} \ll \zeta^{-1}$ by [12; Lemma 30], and so in any case from (140), it follows that on some element $S_{0}$ of $F(-\pi, \pi)$, we have,

$$
\left|M_{3}^{\prime \prime}(\zeta)\right| \leqslant K_{5}|\zeta|^{m / 2-2}|\log \xi|^{s} .
$$

In particular, (141) holds on a set of the form (112) for a suitable $K_{3}$.

For each $r=1,2, \ldots$, we define the disk,

$$
D_{r}:\left|\zeta-\zeta_{r}\right| \leqslant \delta_{2}\left|\zeta_{r}\right|
$$

In view of (136), we have,

$$
|\zeta| \geqslant \frac{1}{2}\left|\zeta_{r}\right| \quad \text { on } D_{r}
$$

and since $\delta_{2}<\operatorname{Sin}\left(c_{2} / 2\right)$, it is clear that for all sufficiently large $r$, say $r \geqslant r_{0}$, the set $D_{r}$ lies in the set (112) on which $v_{3}$ and $v_{4}$ are defined. Finally, we require the following inequalities:

$$
|\zeta|^{(m / 2)-2} \leqslant K_{6}\left|\zeta_{r}\right|^{(m / 2)-2} \text { for all } \zeta \text { in } D_{r},
$$

and if $r$ is sufficiently large, we have

$$
|\log \zeta|^{s} \leqslant K_{7}\left(\log \left|\zeta_{r}\right|\right)^{s} \quad \text { for all } \zeta \text { in } D_{r} .
$$

To prove (144), we obviously have,

$$
\left(1-\delta_{2}\right)\left|\zeta_{r}\right| \leqslant|\zeta| \leqslant\left(1+\delta_{2}\right)\left|\zeta_{r}\right| \quad \text { for } \zeta \text { in } D_{r} \text {. }
$$

If $(m / 2)-2 \geqslant 0$, then (144) follows from the right-hand inequality in (146) since $\delta_{2}<\frac{1}{2}$. If $(m / 2)-2<0$, then (144) follows from the left-hand inequality in (146) since $\delta_{2}<\frac{1}{2}$. 
To prove (145), we need the obvious fact that for $|\zeta| \geqslant \exp \left(\left(\pi^{2} / 3\right)^{\frac{1}{2}}\right)$, we have (147)

$$
\log |\zeta| \leqslant|\log \zeta| \leqslant 2(\log |\zeta|) \text {. }
$$

Thus, if $s \geqslant 0$, it follows from (143), and the right-hand inequalities in (146) and (147) that (145) is valid for all sufficiently large $r$. If $s<0$, we use the left-hand inequalities in (146) and (147) to obtain (145).

Thus, for all sufficiently large $r$, the inequalities (141), (143), (144) and (145) are valid on $D_{r}$ and for each such $r$, we define the function $h_{r}(\zeta)$ by the relation,

$$
G_{r}(\zeta)=G_{r}^{\prime}\left(\zeta_{r}\right)\left(\zeta-\zeta_{r}\right)+h_{r}(\zeta)
$$

so that $h_{r}$ is analytic on $D_{r}$. In view of (135), we clearly have,

$$
h_{r}\left(\zeta_{r}\right)=0, \quad h_{r}^{\prime}\left(\zeta_{r}\right)=0, \quad \text { and } \quad h_{r}^{\prime \prime}(\zeta) \equiv M_{3}^{\prime \prime}(\zeta)
$$

Since (141) holds on $D_{r}$, we have using (144) and (145) that,

$$
\left|h_{r}^{\prime \prime}(\zeta)\right| \leqslant K_{5} K_{6} K_{7}\left|\zeta_{r}\right|^{(m / 2)-2}\left(\log \left|\zeta_{r}\right|\right)^{s} \quad \text { on } D_{r} .
$$

Since $h_{r}^{\prime}\left(\zeta_{r}\right)=0$, and the radius of $D_{r}$ is $\delta_{2}\left|\zeta_{r}\right|$, we thus have,

$$
\left|h_{r}^{\prime}(\zeta)\right| \leqslant K_{5} K_{6} K_{7} \delta_{2}\left|\zeta_{r}\right|^{(m / 2)-1}\left(\log \left|\zeta_{r}\right|\right)^{s} \quad \text { on } D_{r} .
$$

Similarly, since $h_{r}\left(\zeta_{r}\right)=0$, we obtain,

$$
\left|h_{r}(\zeta)\right| \leqslant K_{5} K_{6} K_{7} \delta_{2}^{2}\left|\zeta_{r}\right|^{m / 2}\left(\log \left|\zeta_{r}\right|\right)^{s} \quad \text { on } D_{r} .
$$

But by (130) and (125) (and the fact that $E_{1}\left(\zeta_{r}\right) / 2 N_{s}\left(\zeta_{r}\right) \rightarrow 0$ in (125) as $r \rightarrow+\infty$ ), we have for all sufficiently large $r$ that the inequality $\left|G_{r}^{\prime}\left(\zeta_{r}\right)\right| \geqslant\left|N_{3}\left(\zeta_{r}\right)\right|$ holds, and since $\zeta_{r}$ is a positive real number, we therefore have from (106) that,

$$
\left|G_{r}^{\prime}\left(\zeta_{r}\right)\right| \geqslant\left|K_{2}\right|\left|\zeta_{r}\right|^{(m-2) / 2}\left(\log \left|\zeta_{r}\right|\right)^{s}
$$

From (148), (152) and (153), and the third condition on $\delta_{2}$ in (136), it now follows that if $r$ is sufficiently large, then on the boundary $\left|\zeta-\zeta_{r}\right|=\delta_{2}\left|\zeta_{r}\right|$ of $D_{r}$ we have,

$$
\left|G_{r}(\zeta)\right| \geqslant \delta_{2}\left(\left|K_{2}\right| / 2\right)\left|\zeta_{r}\right|^{m / 2}\left(\log \left|\zeta_{r}\right|\right)^{s}
$$

Now considering the function $E_{3}(\zeta)$ defined in (131), we have by (132) that if we choose a real number $\delta_{3}>0$ such that,

$$
\delta_{3}<\delta_{2} m(2 / 3)^{m / 2} / 8 K_{7}
$$


then there is an element of $F\left(-c_{2}, c_{2}\right)$ on which $\left|E_{3}(\zeta)\right|<\delta_{3}\left|M_{3}(\zeta)\right|$, and so,

$$
\left|E_{3}(\zeta)\right|<\left(4 \delta_{3}\left|K_{2}\right| / m\right)|\zeta|^{m / 2}|\log \zeta|^{s} \text {. }
$$

It now follows from (145), (146) and (155), that if $r$ is sufficiently large, then on $D_{r}$,

$$
\left|E_{3}(\zeta)\right|<\delta_{2}\left(\left|\boldsymbol{K}_{\mathrm{2}}\right| / 2\right)\left|\zeta_{r}\right|^{m / 2}\left(\log \left|\zeta_{r}\right|\right)^{s},
$$

and thus by (154) we have $\left|E_{3}(\zeta)\right|<\left|G_{r}(\zeta)\right|$ on the boundary of $D_{r}$. Since $G_{r}(\zeta)$ has the zero $\zeta_{r}$ in the interior of $D_{r}$, it now follow from Rouche's theorem that equation (133) also has a root $\zeta_{r}^{*}$ in the interior of $D_{r}$. Since equation (133) is equivalent to (129), we see that $\zeta_{r}^{*}$ is a root of $(122)$, and thus $v\left(\zeta_{r}^{*}\right)=0$. From (143), we see that $\left|\zeta_{r}^{*}\right| \rightarrow+\infty$ as $r \rightarrow+\infty$, and since $\zeta_{r}>0$ while $\delta_{2}<\operatorname{Sin} \delta_{1}$, clearly $\zeta_{r}^{*} \operatorname{lies}$ in $|\operatorname{Arg} \zeta|<\delta_{1}$. This proves Lemma $\mathrm{H}$, and concludes the proof of Part (A).

The proof of Part (B) is similar to (but simpler than) the proof of Part (A). We again consider equation (85) which has principal monomials $M_{1}$ and $M_{2}$ given by (88). If $q=0$ and $N \geqslant 1$, the for $1 \leqslant j \leqslant N$, each $\psi_{j}$ is a root of the functions in (89), and $0<\psi_{j}<\pi / n$. As in the proof of Part (A), by using the full strength of [12; Theorem III], we can assert that (85) possesses solutions $u_{1} \sim M_{1}$ and $u_{2} \sim M_{2}$ in $F\left(\psi_{j-1}, \psi_{j-1}\right)$. Choosing a convenient point $c$ on $\operatorname{Arg} z=\psi_{j}$, we then have solutions $w_{k}=\exp \int_{c}^{z} u_{k}$ of $(1)$ on $F\left(\psi_{j_{-1}}, \psi_{j_{+}}\right)$for $k=1,2$, which form a fundamental set. As in Part (A), we show that,

$$
\int_{c}^{z}\left(u_{1}-u_{2}\right)=M_{3}(z)+E_{2}(z) \quad \text { where } E_{2} \ll M_{3} \text { in } F\left(\psi_{j_{-1}}, \psi_{j_{+1}}\right),
$$

and where,

$$
M_{3}(z)=(2 K /(\lambda+2)) z^{(\lambda / 2)+1}
$$

We then consider the equation $w_{1}(z) / w_{2}(z)=s_{1}$, where $s_{1}=\left|s_{1}\right| e^{i m}$ is an arbitrary nonzero complex number. This equation is equivalent to (129) (where $\zeta$ is replaced by $z$ ). Defining $G_{r}(z)$ and $E_{3}(z)$ by (130) and (131), we write (129) in the form (133). Since $\psi_{j}$ is a root of $(89)$, we have,

$$
(\lambda+2) \psi_{j} / 2+\arg K=a \pi / 2,
$$

where $a$ is an odd integer, say $a=2 t+1$. We may assume that $t$ is even, for otherwise the root $-K$ of $K^{2}=-e_{\lambda}$ in (88) can be used in place of $K$. From $(160)$, it follows that the function,

$$
G_{r}(z)=M_{3}(z)-2 \pi i r, \quad \text { for } r=1,2, \ldots,
$$


possesses a root $z_{r}$ on $\operatorname{Arg} z=\psi_{j}$, and that $\left|z_{r}\right| \rightarrow+\infty$ as $r \rightarrow \infty$. As in (148), we define $h_{r}(z)$ by the equation,

$$
G_{r}(z)=G_{r}^{\prime}\left(z_{r}\right)\left(z-z_{r}\right)+h_{r}(z),
$$

and hence,

$$
h_{r}\left(z_{r}\right)=0, \quad h_{r}^{\prime}\left(z_{r}\right)=0, \quad \text { and } \quad h_{r}^{\prime \prime} \equiv M_{3}^{\prime \prime}
$$

We now estimate $h_{r}^{\prime \prime}(z)$ on disks $D_{r}$ of the form $\left|z-z_{r}\right| \leqslant \delta_{2}\left|z_{r}\right|$, where $\delta_{2}$ is a sufficiently small positive number, by using an estimate of the form (144). It follows exactly as in the proof of Part (A) that if $r$ is sufficiently large, then $\left|E_{3}(z)\right|<\left|G_{r}(z)\right|$ on the boundary of $D_{r}$, and so the equation $w_{1}(z) / w_{2}(z)=s_{1}$ has a root $z_{r}^{*}$ in $D_{r}$, with the asserted exponent. Since $\delta_{2}$ can be chosen so small that the disks $D_{r}$ eventually lie in $\psi_{j}-\varepsilon_{1}<\Delta \operatorname{\Delta rg} z<\psi_{i}+\varepsilon_{1}$, the proof of Part (B) is complete.

7. - REMARK. - From the proof of Part (A) of Theorem 3, it is clear that the solutions $w_{1}$ and $w_{2}$ which were constructed, each have no zeros on the set $E$ defined by (115) and (116), since from (111), the functions $v_{3}$ and $v_{4}$ have no zeros on the set (112). Similarly, it is clear from the proof of Part (B), that the solutions $w_{1}$ and $w_{2}$ which were constructed, have only finitely many zeros on a sector of the form $\psi_{j}-\varepsilon_{2}<\operatorname{Arg} z<\psi_{i}+\varepsilon_{2}$ for some $\varepsilon_{2}>0$.

8. - Remark. - In the case of an equation (1) (where $A(z)$ has the form (2)) which possesses a fundamental set $w_{1}^{*}, w_{2}^{*}$, each having no zeros (and such equations do exist for any given $P(z)$ (see $[6 ; \S 10])$ ), it is clear that the solutions $w_{1}$ and $w_{2}$ constructed in Theorem $3(A)$ for a given $a_{j_{p}}$ must be constant multiples of $w_{1}^{*}$ and $w_{2}^{*}$. (This follows easily from the property of $w_{1}$ and $w_{2}$ stated in Theorem $3(A)$.) Hence, in this case, any linear combination $c w_{1}^{*}+c_{0} w_{2}^{*}$, where the complex constants $c$ and $c_{0}$ are both nonzero, must have infinitely many zeros around each of the special curres $\operatorname{Im} P(z)=a_{i_{s}}$ (in the sense given in Theorem $3(A)$ ). (The equation (21) provides the simplest example of such an equation where $A(z)$ is transcendenta1.)

\section{REFERENCES}

[1] S. BANK, On the value-distribution theory for entire solutions of second-order linear differential equations, Proc. London Math. Soc., (3) 50 (1985), pp. 505-534.

[2] S. Bank, On the instability theory of differential polynomials, Ann. Mat. Pura Appl., 74 (1966), pp. 83-112. 
[3] S. BANK, On solutions having large rate of growth for nonlinear differential equations in the complex domain, J. Math. Anal. Appl., 22 (1968), pp. 129-143.

[4] S. BANK, On the location of complex zeros of solutions of periodic differential equations, Applicable Analysis, 22 (1986), pp. 111-132.

[5] S. Bank - I. LaIne, On the oscillation theory of $f^{\prime \prime}+A f=0$ where $A$ is entire, Trans. Amer. Math. Soc., 273 (1982), pp. 351-363.

[6] S. BANK - I. LAINE - J. LANGLEY, On the frequency of zeros of solutions of second-order linear differential equations, Resultate der Math., 10 (1986), pp. 8-24.

[7] S. BanK - J. Languey, On the oscillation of solutions of certain linear differential equations in the complex domain, Proc. Edinburgh Math. Soc., 30 (1987), pp. 455-469.

[8] E. Hituf, On the zeros of Mathieu functions, Proc. London Math. Soc., (2) 23 (1924), pp. 185-237.

[9] E. HrLce, Ordinary Differential Equations in the Complex Domain, Wiley, New York, 1976.

[10] E. Hille, Lectures on Ordinary Differential Equations, Addison-Wesley, Reading, Mass., 1969.

[11] R. Nevandinna, Über Riemannsche Flächen mit endlich vielen Windungspunkten, Acta Math., 58 (1932), pp. 295-373.

[12] W. STRODт, Contributions to the asymptotic theory of ordinary differential equations in the complex domain, Mem. Amer. Math. Soc., No. 13 (1954).

[13] W. Strodt, Principal solutions of ordinary differential equations in the complex domain, Mem. Amer. Math. Soc., No. 26 (1957).

[14] W. StRoDt, On the algebraic closure of certain partially ordered fields, Trans. Amer. Math. Soc., 105 (1962), pp. 229-250.

[15] W. Strodt - R. WRight, Asymptotic behavior of solutions and adjunction fields for nonlinear first order differential equations, Mem. Amer. Math. Soc., No. 109 (1971).

[16] H. W Iтт1сн, Eindeutige Lösungen der Differentialgleichung $w^{\prime}=R(z, w)$, Math. Z., 74 (1960), pp. 278-288. 